\title{
Action potentials of HL-1 cells recorded with silicon nanowire transistors
}

Jan Felix Eschermann, Regina Stockmann, Martin Hueske, Xuan Thang Vu, Sven Ingebrandt, and Andreas Offenhäusser

Citation: Appl. Phys. Lett. 95, 083703 (2009);

View online: https://doi.org/10.1063/1.3194138

View Table of Contents: http://aip.scitation.org/toc/apl/95/8

Published by the American Institute of Physics

\section{Articles you may be interested in}

Organic electrochemical transistors for cell-based impedance sensing

Applied Physics Letters 106, 043301 (2015); 10.1063/1.4906872

VAPOR-LIQUID-SOLID MECHANISM OF SINGLE CRYSTAL GROWTH

Applied Physics Letters 4, 89 (1964); 10.1063/1.1753975

High-transconductance graphene solution-gated field effect transistors

Applied Physics Letters 99, 033503 (2011); 10.1063/1.3614445

High speed and high density organic electrochemical transistor arrays

Applied Physics Letters 99, 163304 (2011); 10.1063/1.3652912

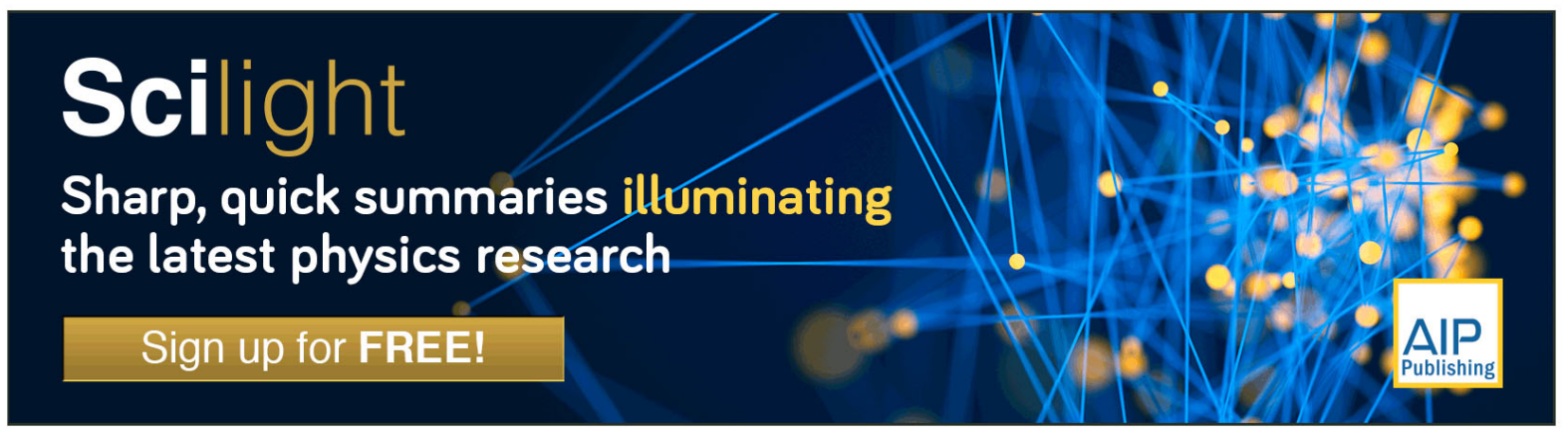




\title{
Action potentials of HL-1 cells recorded with silicon nanowire transistors
}

\author{
Jan Felix Eschermann, ${ }^{1,2}$ Regina Stockmann, ${ }^{1,2}$ Martin Hueske, ${ }^{1,2}$ Xuan Thang Vu, ${ }^{1,2}$ \\ Sven Ingebrandt, ${ }^{1,2,3}$ and Andreas Offenhäusser ${ }^{1,2, a)}$ \\ ${ }^{1}$ Institute of Bio- and Nanosystems (IBN2), Forschungszentrum Jülich GmbH, 52425 Jülich, Germany \\ ${ }^{2}$ Jülich-Aachen Research Alliance - Fundamentals of Future Information Technologies (JARA-FIT), \\ 52425 Jülich, Germany \\ ${ }^{3}$ Informatics and Microsystems Technology, University of Applied Sciences Kaiserslautern, \\ 66482 Zweibrücken, Germany
}

(Received 12 March 2009; accepted 13 July 2009; published online 27 August 2009)

\begin{abstract}
Silicon nanowire (NW) transistors were fabricated in a top-down process. These devices were used to record the extracellular potential of the spontaneous activity of cardiac muscle HL-1 cells. Their signals were measured by direct dc sampling of the drain current. An improved signal-to-noise ratio compared to planar field-effect devices was observed. Furthermore the signal shape was evaluated and could be associated to different membrane currents. With these experiments, a qualitative description of the properties of the cell-NW contact was obtained and the suitability of these sensors for electrophysiological measurements in vitro was demonstrated. (C) 2009 American Institute of Physics. [DOI: 10.1063/1.3194138]
\end{abstract}

In recent years, nanoscale features have played an increasingly important role in the development of highly sensitive biosensors. Silicon nanowires $(\mathrm{NWs})^{1-5}$ have become particularly interesting due to their high sensitivity, selectivity when functionalized, and compatibility with mature semiconductor technologies. While a growing number of studies have demonstrated the capability of detecting molecules, viruses, or antibodies, hardly any studies were able to measure the behavior of cells with this kind of technology. So far, the coupling of NWs to cells has been demonstrated once with neuronal cells ${ }^{6}$ and in a very recent publication with cardiomyocytes and aortic smooth muscle cells. ${ }^{7}$ However, the signal shapes in these studies were different from previous recordings with planar transistors and so far are not well understood.

Here, we present extracellular recording of HL-1 cells' electrical activity with NW transistors and analyze the physiological relevance of the obtained data. HL-1 cells are a cardiac muscle cell line derived from a mouse atrial cardiomyocyte tumor. ${ }^{8}$ This cell type is well suited for our studies in two respects: (i) the cell line is robust and well understood, which allows convenient studies of the fundamental physics of the coupling and (ii) it is closely related to cardiomyocytes which have been used for in vitro pharmacological studies. ${ }^{910}$ We compare our signals with ones from planar field-effect transistors (FETs) $)^{10,11}$ and associate them with the point-contact model ${ }^{12}$ in order to explain their shape physiologically. This analysis is important to understand how to associate the recorded signals to the cellular mechanisms generating them. Only in this manner it will be possible to exploit the increased sensitivity of these devices in pharmaceutical studies such as locally resolved examination of ion channel dynamics.

The NW field-effect transistors were fabricated in a top-down approach using nanoimprint lithography and anisotropic wet silicon etching with tetramethylammonium hydroxide (TMAH). ${ }^{13-16}$ Using this approach we ensured a

\footnotetext{
a) Author to whom correspondence should be addressed. Electronic mail: a.offenhaeusser@fz-juelich.de.
}

high reproducibility, scalability and compatibility with standard complementary metal oxide semiconductor (CMOS) technology. ${ }^{2}$ The devices were fabricated on 4 in. silicon-on-insulator wafers with boron doping $\left(1 \times 10^{15} \mathrm{~cm}^{-3}\right)$. In the beginning, the device layer was thinned to $60 \mathrm{~nm}$ by successive thermal oxidation and wet chemical etching with buffered hydrofluoric acid. In the last thinning step, a $100 \mathrm{~nm} \mathrm{SiO}{ }_{2}$ layer was left on top and structured with nanoimprint lithography in combination with reactive ion etching. Thus a hard mask was obtained, which protected the feed lines and the NWs from the subsequent TMAH etching resulting in wires with trapezoidal cross section. Afterwards, the feed lines were boron doped with an energy of $7 \mathrm{keV}$ and a dose of $10^{14} \mathrm{~cm}^{-2}$ resulting in an impurity concentration of approximately $10^{19} \mathrm{~cm}^{-3}$ in order to reduce the serial resistance. Then, a $280 \mathrm{~nm}$ thick lowpressure chemical vapor depostion (LPCVD) $\mathrm{SiO}_{2}$ was deposited and structured by photolithography such that only the NWs were exposed during thermal gate oxide growth (thickness of $8 \mathrm{~nm}$ ). This step was followed by structuring metal contacts by lift-off. Finally, the chips were wire bonded and encapsulated with polydimethylsiloxane (PDMS) (Dow Corning, Product No. 96-083) and a glass ring (diameter of $16 \mathrm{~mm}$ ) forming a small cavity for cell culture. On each chip there are $4 \times 4$ recording sites $\left(12 \times 8 \mu \mathrm{m}^{2}\right)$ with a pitch of $200 \mu \mathrm{m}$, each consisting of six parallel wires with widths of $500 \mathrm{~nm}$ (mold dimension, i.e., due to underetching the actual size was approximately $350 \mathrm{~nm}$ at the bottom of the wire). This design was chosen in order to enable a recording from single cells which typically are around $20 \mu \mathrm{m}$ in diameter. A representative active area is shown in Fig. 1.

HL-1 cells $\left(10^{5}\right.$ cells $\left./ \mathrm{cm}^{2}\right)$ were plated on fibronectin coated chips 3 days prior to the experiment and cultured in a supplemented Claycomb Medium ${ }^{8}$ (JRH Biosciences). The supplements were $10 \%(\mathrm{~V})$ fetal bovine serum, $100 \mathrm{U} / \mathrm{ml}$ penicillin, $100 \mu \mathrm{g} / \mathrm{ml}$ streptomycin, $0.1 \mathrm{mM}$ norepinephrine (NE) and $2 \mathrm{mM} \mathrm{L}$-glutamine. This medium was exchanged every day and $3 \mathrm{~h}$ before the experiment. On the day of experiment, the cells had formed a confluent layer on top of the chip as shown in Fig. 2. It is known that HL-1 cells show 


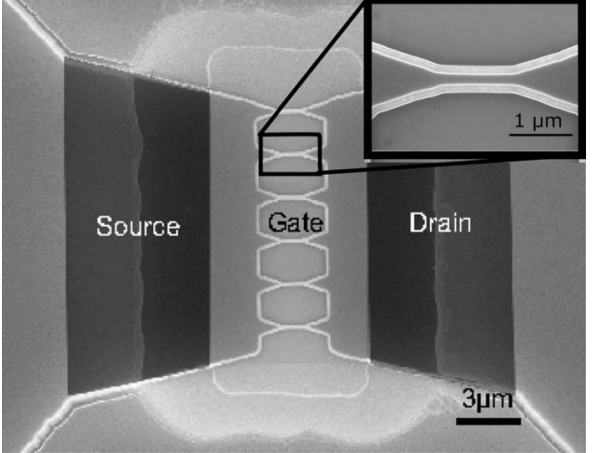

FIG. 1. Layout of one NW transistor consisting of six wires in parallel. The image shows an SEM picture with overlaid highlighting of source, drain and gate areas. A closeup of one single NW can be seen in the inset.

spontaneous electrical activity in the presence of $0.1 \mathrm{mM}$ NE. ${ }^{8}$ This activity can readily be recorded without prior stimulation. Furthermore, signal propagation across the culture is known to occur via cell-cell gap junctions.

For data readout a custom-made 16 channel impedance converter was connected to a measurement card (National Instruments, PCI-6071E). This setup can be used to record transistor characteristics as well as sample time series with a rate of $10 \mathrm{ksamples} / \mathrm{s} .{ }^{16}$ We chose such a setup in contrast to the often used lock-in technique ${ }^{1,3,6}$ in order to observe the full spectrum of the signals (up to $5 \mathrm{kHz}$ ). Furthermore, we observed signal distortions with the lock-in technique at frequencies as low as $50 \mathrm{~Hz}$ due to parasitic capacitances of the conducting lines on the chip. This distortion makes a proper interpretation of the recorded signals difficult. Simultaneous sampling from 16 channels also enabled monitoring the signal propagation across the whole chip in a single measurement (see supplementary information). ${ }^{18}$ The operating point was chosen such that the transconductance $g_{m}=d I_{\mathrm{d}} / d V_{\mathrm{gs}}$ is maximum, where $I_{\mathrm{d}}$ is the current through the drain contact and $V_{\mathrm{gg}}$ is the potential difference between an $\mathrm{Ag} / \mathrm{AgCl}$ wire immersed into the electrolyte and the source contact. In the measurements presented here, that was the case for a drainsource voltage $V_{\mathrm{ds}}=-2.0 \mathrm{~V}$ and $V_{\mathrm{gs}}=-0.75 \mathrm{~V}$ (i.e., the reference electrode is at the more negative potential) resulting in $I_{\mathrm{d}}=3 \pm 1 \mu \mathrm{A}$ and $g_{m}=4.5 \pm 0.5 \mu \mathrm{S}$. Under these operating conditions, continuous measurements were possible for up to approximately two hours until the cells died. A degradation of the chips could not be observed. However, there was a considerable amount of drift in our system. It is known that $\mathrm{SiO}_{2}$ gate dielectrics are unstable in an electrolyte

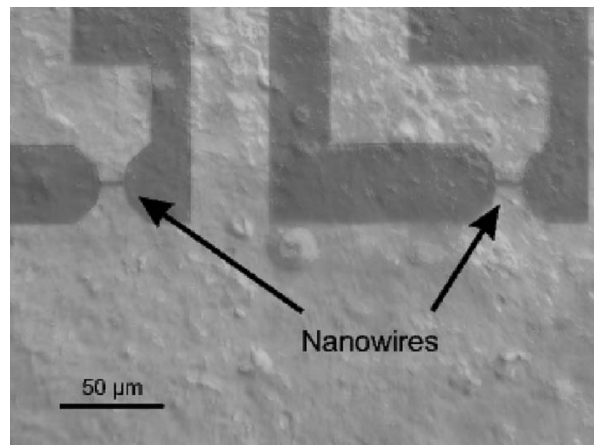

FIG. 2. Chip surface with HL-1 cells on top. The cells form a dense monolayer appearing like a rough surface.
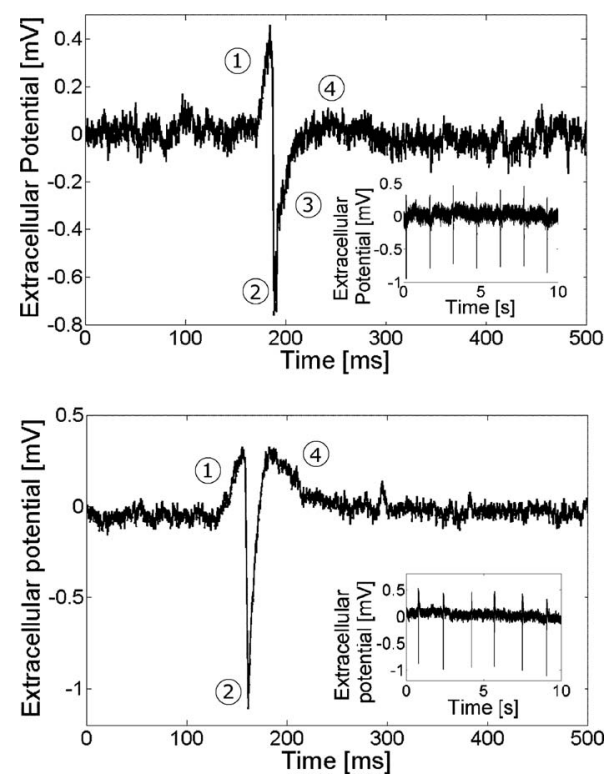

FIG. 3. Extracellular recordings from two different chips showing the different signal components. The insets show ten seconds of the same recordings.

environment, ${ }^{17}$ which is the main reason for this observation. Still, our measurements could be stabilized by controlling the temperature and using a standard reference electrode which would be more robust against leakage currents through the passivation.

Figure 3 shows action potentials recorded from different cell passages and different chips. These recordings are representative of all the observed signal shapes (two passages with approximately 30 transistors on three chips). Both traces share a positive peak (amplitude about $0.4 \mathrm{mV}$, duration $8 \mathrm{~ms}$ ) in the beginning (1) followed by a negative peak $(1.0 \mathrm{mV}, 2 \mathrm{~ms})$ (2). Afterwards, however, the upper trace has an almost invisible plateau (3) and a subsequent broad $(100 \mathrm{~ms})$ and flat positive hump (4) while the lower trace does not have a plateau but a much more pronounced second positive peak $(80 \mathrm{~ms})(4)$. Similar traces were also recorded with planar FETs (e.g., Fig. 4). Additionally, the signal propagation across the cell layer has been monitored and is discussed in the supplementary material. ${ }^{18}$

Previous studies have discussed several different signal shapes of extracellular recordings in detail. ${ }^{10,11}$ These studies found that peaks like (1) and (2) were present in all cases while differences were observed for the latter, slower components (3) and (4), as in our study. Sprössler et $a .^{11}$ simulated the recorded signals by evaluating the

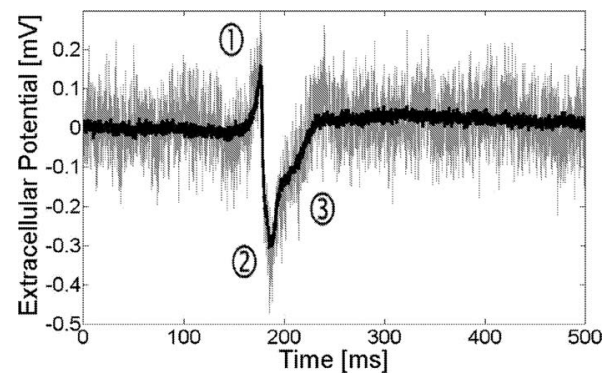

FIG. 4. Representative extracellular recording of an HL-1 action potential with a planar FET (black: average of 57 action potentials, gray: single trace). The numbering is used in accordance to Fig. 3. 
point-contact model for different ion permeabilities. Components (1)-(4) could be associated with their physiological origin by augmenting cell behavior using pharmacological agents. ${ }^{10}$ The positive peak was caused by a stimulus current injected via gap junctions from neighboring cells. By this initial depolarization, an opening of the voltage-gated sodium channels was induced. Consequently, the negative overshooting spike (2) can be related to an inward directed $\mathrm{Na}^{+}$current, which is followed by an inward current of $\mathrm{Ca}^{2+}$ ions to maintain the depolarization causing a plateau (3). Finally, the cell is repolarized by a flow of $\mathrm{K}^{+}$out of the cell. Deviations from this ideal time course can be attributed to many factors such as differences in coupling to the transistor (e.g., due to partial coverage) or variable intensities of $\mathrm{Ca}^{2+}$ or $\mathrm{K}^{+}$currents in the channel region ${ }^{10}$ (e.g., due to different ion channel expression in each passage).

Although we did not perform extensive pharmaceutical tests, we examined the effect of the potassium-selective ion channel blocker tetraethylammonium (TEA) on the recorded signals and verified the expected time-dependent decrease in signal amplitude. ${ }^{18}$ However, for safety pharmacology it would be desirable to record the $T$ wave peak of cellular signals, which cannot be seen in our measurements. In order to achieve this goal we need to reduce the drift and the noise which we observed, e.g., by using a better reference electrode.

As all observations can be explained in terms of previously published data from planar FETs, the hypothesis seems justified that recordings with such devices and NWs in principle are comparable and that the point contact model is suitable for NW devices, too. However, it is remarkable that the signal-to-noise ratio in the recordings presented here is much higher than for standard open gate transistors (see Fig. 4). The gate-referred noise for planar FETs typically ranges between 100 and $200 \mu \mathrm{V}^{19,20}$ (rms value). The lowest value of $85 \mu \mathrm{V}$ (calculated from the peak to peak value) was reported by Offenhäusser et $a l^{21}$ In contrast, our NW transistors have a minimum value of $34 \mu \mathrm{V}$ (mean: $47 \mu \mathrm{V} \pm 15 \mu \mathrm{V}, N=16)$. We attribute this to the fact that the TMAH etching step resulted in surfaces with few defects. However, multi electrode arrays are still better in this respect. We did not include them in our comparison as they rely on a completely different technology. But our signals are also stronger which could be attributed to a better sealing of the cells on the nanostructured surface.

In this study we have shown measurements of the electrical activity of HL-1 cells with NW transistors arranged in a two dimensional array. By comparing the data with previously published traces we could identify the different parts of the signal and relate them to the activity of certain types of ion channels. This analysis shows that the coupling to NW sensors is physically comparable to the coupling with planar devices. Remarkably, we were successful with direct impedance converter readout of the signals. Using this method is an improvement over data obtained by lock-in measurements since the cellular activity can be monitored with its full bandwidth and signal distortions due to parasitic capacitances can be precluded. Still, the signal-to-noise ratio is higher for our system than for standard planar devices indicating that measurements with neuronal cells without averaging should be possible. This result opens further possibilities for our technology such as studying the network behavior of large cultures of neurons with a highly integrated version of our system consisting of $32 \times 32$ recording sites with a pitch of $50 \mu \mathrm{m}$ in each direction. ${ }^{13}$ At the moment, however, we are focusing on scaling aspects of the NWs and examining the coupling quantitatively in experiments with patch-clamp control.

We thank the German Research Foundation for the financial support through the project "Mi-Besan" (contract no. OF22/7-1). Furthermore, we are grateful for the support during chip production of K.-H. Deussen, W. Michelsen, (both IBN1, FZJ), and the IBN process technology group. Also our thanks go to R. Helpenstein and B. Hofmann (both IBN2$\mathrm{BE}$ ) for their help with cell culturing. K. E. Michael is acknowledged for her help in the preparation of the manuscript.

${ }^{1}$ Y. Cui, Q. Wei, H. Park, and C. M. Lieber, Science 293, 1289 (2001).

${ }^{2}$ E. Stern, J. F. Klemic, D. A. Routenberg, P. N. Wyrembak, D. B. TurnerEvans, A. D. Hamilton, D. A. LaVan, T. M. Fahmy, and M. A. Reed, Nature (London) 445, 519 (2007).

${ }^{3}$ A. Kim, C. S. Ah, H. Y. Yu, J.-H. Yang, I.-B. Baek, C.-G. Ahn, C. W. Park, M. S. Jun, and S. Lee, Appl. Phys. Lett. 91, 103901 (2007).

${ }^{4}$ G. B. Kang, J. M. Park, S. G. Kim, J. G. Koo, J. H. Park, Y.-S. Sohn, and Y. T. Kim, Electron. Lett. 44, 1876 (2008).

${ }^{5}$ P. R. Nair and M. A. Alam, Appl. Phys. Lett. 88, 233120 (2006)

${ }^{6}$ F. Patolsky, B. P. Timko, G. Yu, Y. Fang, A. B. Greytak, G. Zheng, and C. M. Lieber, Science 313, 1100 (2006).

${ }^{7}$ T.-S. Pui, A. Agarwal, F. Ye, N. Balasubramanian, and P. Chen, Small 5, 208 (2009).

${ }^{8}$ W. C. Claycomb, N. A. Lanson, Jr., B. S. Stallworth, D. B. Egeland, J. B. Delcarpio, A. Bahinski, and N. J. Izzo, Jr., Proc. Natl. Acad. Sci. U.S.A 95, 2979 (1998).

${ }^{9}$ C.-K. Yeung, S. Ingebrandt, M. Krause, A. Offenhäusser, and W. Knoll, J. Pharmacol. Toxicol. Methods 45, 207 (2001).

${ }^{10}$ S. Ingebrandt, C.-K. Yeung, M. Krause, and A. Offenhäusser, Biosens. Bioelectron. 16, 565 (2001).

${ }^{11}$ C. Sprössler, M. Denyer, S. Britland, W. Knoll, and A. Offenhäusser, Phys. Rev. E 60, 2171 (1999).

${ }^{12}$ W. G. Regehr, J. Pine, C. S. Cohan, M. D. Mischke, and D. W. Tank, J. Neurosci. Methods 30, 91 (1989).

${ }^{13}$ J.F. Eschermann, X.T. Vu, R. Stockmann, T. Dufaux, A. Offenhäusser, and S. Ingebrandt, Book of Abstracts of the Nanoelectronics Days (unpublished).

${ }^{14}$ X. T. Vu, J. F. Eschermann, R. Stockmann, R. GhoshMoulick, A. Offenhäusser, and S. Ingebrandt, Phys. Status Solidi A 206, 426 (2009).

${ }^{15}$ X. T. Vu, R. Ghosh Moulick, J. F. Eschermann, R. Stockmann, A. Offenhäusser, and S. Ingebrandt, "Fabrication and Application of Silicon Nanowire Transistor Arrays for Biomolecular Detection," Sens. Actuators B (to be published).

${ }^{16}$ J. F. Eschermann, R. Stockmann, X. T. Vu, T. Dufaux, A. Offenhäusser, and S. Ingebrandt, Technical Digest of 19th MicroMech E. Workshop, 2008 (unpublished).

${ }^{17}$ T. Matsuo and M. Esashi, Sens. Actuators 1, 77 (1981).

${ }^{18}$ See EPAPS supplementary material at http://dx.doi.org/10.1063/ 1.3194138 for signal propagation across the cell layer.

${ }^{19}$ A. Offenhäusser, C. Sprössler, M. Matsuzawa, and W. Knoll, Biosens. Bioelectron. 12, 819 (1997).

${ }^{20}$ S. Vassanelli and P. Fromherz, Appl. Phys. A: Mater. Sci. Process. 66, 459 (1998)

${ }^{21}$ A. Offenhäusser, J. Rühe, and W. Knoll, J. Vac. Sci. Technol. A 13, 2606 (1995) 